\title{
A 3-D-printable plastic trough for serial semithin sectioning
}

\author{
Viktor Starunov ${ }^{1,2}$ \\ ${ }^{1}$ Department of Invertebrate Zoology, Saint Petersburg State University, \\ Universitetskaya nab., 7-9, 199034, Saint Petersburg, Russian Federation \\ 2Zoological Institute RAS, Universitetskaya nab. 1, 199034, Saint Petersburg, \\ Russian Federation \\ Correspondence and requests for materials should be addressed to Viktor Starunov, \\ starunov@gmail.com
}

\begin{abstract}
A simple lightweight 3-D-printable trough to make ribbons of semithin sections is described. The trough is large enough to fit the standard glass slide. The sectioning process is identical to a diamond histo jumbo knife; therefore, the trough may be recommended for specimens which contain hard particles that could damage the knife. The trough is designed to fit the three most common glass knife sizes and can be used with different ultratome models.
\end{abstract}

Keywords: 3-D reconstruction, 3-D printing, microscopy, semithin sectioning, glass knife, trough.

\section{Introduction}

The three-dimensional volume reconstruction methods based on a series of histological sections are now between state-of-the-art tools of modern biology, such as confocal microscopy and microtomography (Ruthensteiner, 2008; Handschuh, Schwaha and Metscher, 2010). They are widely used in zoology, neurobiology, developmental biology and other fields (Böhm, Szucsich and Pass, 2012; Leys, Kamarul Zaman and Boury-Esnault, 2016; Roth, Wilson and Sandig, 2015; Tilic et al., 2015). The volume reconstruction requires a complete series of paraffin- or resin-embedded histological sections. Resin sections in small specimens offer a larger amount of information compared to the conventional paraffin sections because of higher section quality and lesser section thickness (Ruthensteiner, 2008).

The invention of the diamond Jumbo knife allowed the significant simplification of the process of serial semithin sectioning (Blumer et al., 2002). Different techniques were elaborated to use the Jumbo knives (Handschuh, Schwaha and Metscher, 2010; Lucas et al., 2012; Pernstich, Krenn and Pass, 2003; Schoenwolf and Chandler, 1983). The use of a diamond knife is almost ideal for most purposes, but nevertheless, there are numerous specimens that can contain hard particles, such as sand or mineralized skeletal elements, that may seriously damage the knife. This is especially the case in burrowing or sediment-feeding annelids, or sessile animals like sponges or bryozoans. For such objects glass knives may be used instead of diamond, since they are cheap, disposable, and work fine with semithin sections. However, the commercially available troughs are designed for ultrathin or single semithin sections only. There are several designs of specialized trough, which allow work with ribbons of semithin sections (Campbell, 1981; Royer, 1988; Ruthensteiner, 2008; Wali and Jagadeesh, 1988), but in most cases, they are not easy to make and/or do not allow the use of regular $26 \times 76 \mathrm{~mm}$ glass slides as easily as a diamond Jumbo knife.

Nowadays the widespread distribution of 3-D printing provides the possibility to easily make plastic parts of complex geometry with high accuracy. They also make it possible to multiply designed objects and modify already existing 
models. The wide variety of available filament materials makes it possible to adjust the properties of the resulting parts for different uses. In this article I describe a lightweight, easy to make and inexpensive 3 -D-printed plastic trough, designed to use with standard glass knives that can be used as a substitution for a diamond Jumbo knife for complicated samples.

\section{Methods}

The modelling process was performed using Autodesk Tinkercad platform (Autodesk, San Rafael, California, USA). The troughs were developed for the three most common knife sizes: $6.4 \mathrm{~mm}, 8 \mathrm{~mm}$, and $10 \mathrm{~mm}$. The slicing of the exported STL files was done with Ultimaker Cura 3.6.0 (Ultimaker, Geldermalsen, Netherlands) with the following settings: Layer height $-0.06-0.1 \mathrm{~mm}$; Wall thickness - $1.2 \mathrm{~mm}$; Top/Bottom thickness $1.2 \mathrm{~mm}$; Infill density - 10-25\%; Print speed - up to $40 \mathrm{~mm} / \mathrm{sec}$. Build plate adhesion type - brim. I tested horizontal and vertical model orientations. While printing in vertical orientation does not need any support, for horizontal orientation the support generating should be applied.

The printing was performed using Anycubic I3 mega instrument (Anycubic, China). Different filament types were tested: Acrylonitrile butadiene styrene (ABS), Styrene-butadiene-styrene polymer (SBS), Polyethylene terephthalate glycol-modified (PET-G), Polylactic acid (PLA), and High-impact polystyrene (HIPS). The temperature settings for the extruder and build plate were applied according filament manufacturer's instructions. The post-processing was applied when needed. To smooth the models a short rinsing in chloroform with subsequent drying under a fume hood for 3-12 hours was performed. The knife fastening was done with an M3 screw and a hexagonal nut. Afterward, the trough was sealed with hot melted pink dental wax.

Specimens of different annelid species were used to test the trough. The animal samples were fixed in $2.5 \%$ glutaraldehyde in $0.1 \mathrm{M}$ phosphate buffer $(\mathrm{PB}, \mathrm{pH}=7.2)$ with $0.3 \mathrm{M} \mathrm{NaCl}$, rinsed one to three times in $\mathrm{PB}$ with $0.3 \mathrm{M} \mathrm{NaCl}$ and postfixed in $1 \% \mathrm{OsO} 4$ in the same buffer. The dehydration was performed in acetone series of increasing concentration. Afterwards the specimens were embedded in Epon-Araldite. The sectioning was performed using Reichert Ultracut E (Reichert, Austria)

\section{Results and discussion}

The general design of the trough is shown in Figure 1. The STL model files for three different knife sizes are available at the Thingiverse website (https://www.thingiverse.com/thing:3427208). The trough was designed to work with Reichert and Leica instruments. However, it
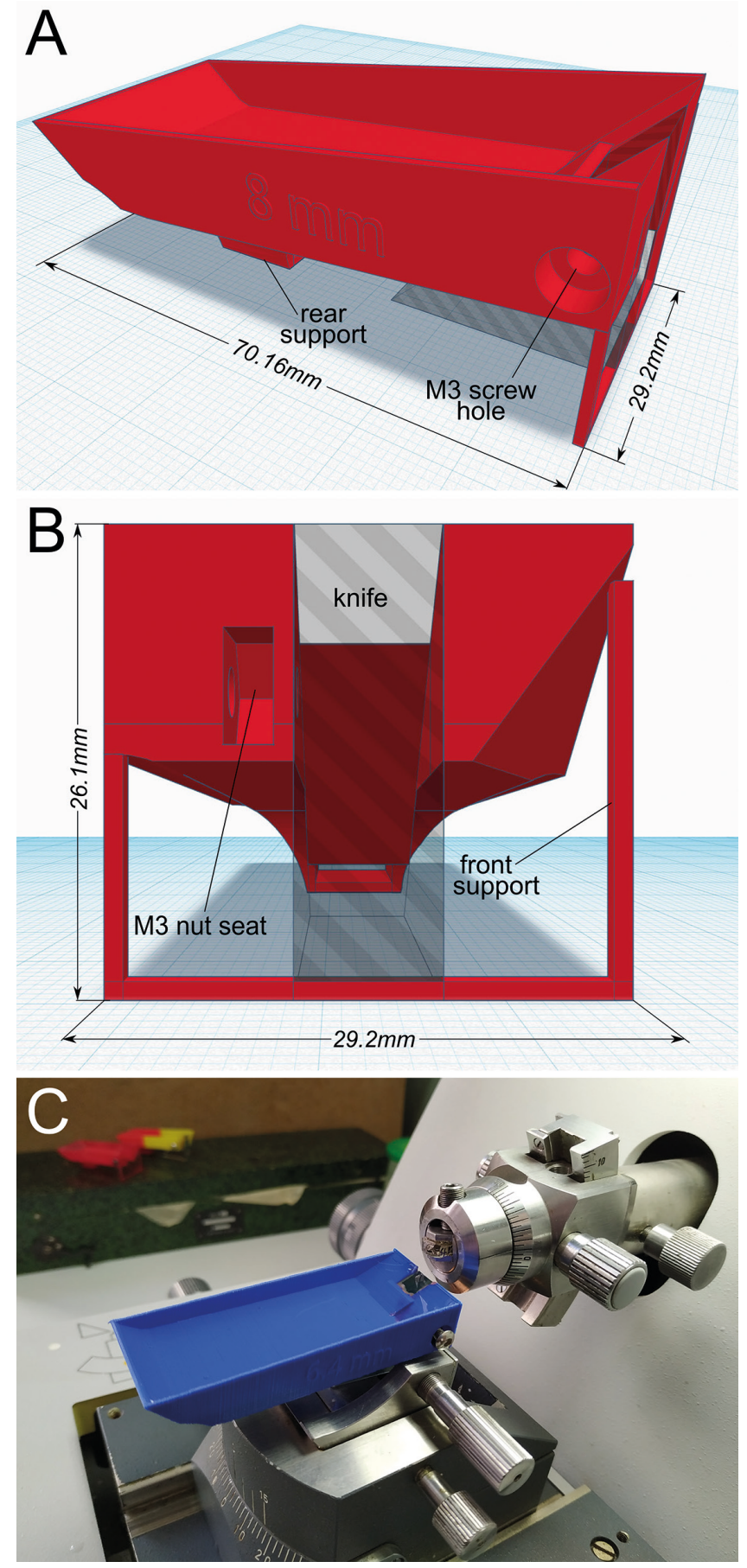

Fig. 1. A general design of the trough $(A, B)$ and a working prototype made of PLA plastic while sectioning the epoxy resin embedded block (C).

is compatible with virtually all types of ultratomes, and easily can be re-modelled for any type of mount. The trough is $70.2 \mathrm{~mm}$ long and $29.2 \mathrm{~mm}$ wide. The inner dimensions are: $69 \mathrm{~mm}$ length, $27 \mathrm{~mm}$ width and up to $9 \mathrm{~mm}$ depth. The water capacity of the trough is about $12.5 \mathrm{ml}$. The flat part of the bottom is $50 \mathrm{~mm}$ long to fit standard $24 \times 50 \mathrm{~mm}$ cover glass. Since the 3 -D-printer does not make ideally flat surfaces, I recommend putting 
the cover glass at the bottom of the trough. It is important to improve the gliding of the slide edge while collecting the ribbons. The frontal side of the trough has supports that are necessary for stability when printing in an upright position and should be removed before knife installation. There is also a rear support to make the trough more sustainable when the knife is attached. The weight of the trough is from six to nine g depending on material and infill density.

To facilitate knife fastening and make the junction more solid, the trough has a hole for a $10 \mathrm{~mm}$ long M3 screw at the right side and a seat for a standard hexagonal M3 nut at the frontal face. The knife should be mounted flush to the front face of the trough. The sealing can be done with dental wax or nail polish. After work is completed, the wax can be cleared mechanically. I also recommend sealing the screw hole to minimize the risk of water leakage. Since the recommended clearance angle for the glass knife is $5-6^{\circ}$, the top face of the trough is beveled to compensate for its inclination in the working position.

All types of tested filament were found to be convenient for the trough. However, I would recommend PLA plastic since it is easier to use and produces fewer pollutants during printing (Stephen et al., 2013), biodegradable (Fukushima et al., 2009; Qi, Ren, Wang, 2017), and practically non-soluble in acetone. The last feature allows washing away the sealing and makes possible multiple uses of nail polish as a sealing agent. I use the trough without any post-processing, however, to make it smoother one can treat it with chloroform or ethyl acetate. The color of the plastic does not matter, however, in my opinion, dark colors such as black or dark blue are preferable since they provide more contrast for work with single slices as well as ribbons.

Operation of the printed trough is identical to those with diamond Jumbo knife (Blumer et al., 2002; Ruthensteiner, 2008). Therefore, it is possible to use the trough in the education process, for training before using the diamond knife. Another advantage of the trough is the simple switch from diamond to glass knife without any changes in the sectioning process, which is important for complicated specimens with a high risk of knife damaging.

To summarize, a 3-D-printable trough is a cheap and easy-to-use alternative to a diamond Jumbo knife. Its monolithic construction is fairly leak-proof and can be re-used multiple times. The provided model can be easily reproduced using most of the common 3-D printers in different types of plastic and may be modified to adopt any special needs.

\section{Acknowledgements}

The development of the trough was performed at the Center for Culturing Collection of Microorganisms, and center "CHROMAS" of St. Petersburg State University.

\section{References}

Blumer, M.J.F., Gahleitner, P., Narzt, T., Handl, C., Ruthensteiner, B., 2002. Ribbons of semithin sections: an advanced method with a new type of diamond knife. Journal of Neuroscience Methods 120:11-16. https://doi. org/10.1016/S0165-0270(02)00166-8

Böhm, A., Szucsich, N. U., Pass, G., 2012. Brain anatomy in Diplura (Hexapoda). Frontiers in Zoology 9:1-17. https:// doi.org/10.1186/1742-9994-9-26

Campbell, R. D., 1981. A method for making ribbons of semithin plastic sections. Biotechnic \& Histochemistry 56:247249. https://doi.org/10.3109/10520298109067319

Fukushima, K., Abbate, C., Tabuani, D., Gennari, M., Camino, G., 2009. Biodegradation of poly(lactic acid) and its nanocomposites. Polymer Degradation and Stability 94:1646-1655. https://doi.org/10.1016/j.polymdegradstab.2009.07.001

Handschuh, S., Schwaha, T., Metscher, B. D., 2010. Showing their true colors: A practical approach to volume rendering from serial sections. BMC Developmental Biology 10. https://doi.org/10.1186/1471-213X-10-41

Leys, S. P., Kamarul Zaman, A., Boury-Esnault, N., 2016. Three-dimensional fate mapping of larval tissues through metamorphosis in the glass sponge Oopsacas minuta. Invertebrate Biology 135:259-272. https://doi.org/10.1111/ivb.12142

Lucas, M. S., Günthert, M., Gasser, P., Lucas, F., Wepf, R., 2012. Bridging Microscopes: 3D Correlative Light and Scanning Electron Microscopy of Complex Biological Structures. Methods in Cell Biology 111:325-356. https://doi. org/10.1016/B978-0-12-416026-2.00017-0

Pernstich, A., Krenn, H.W., Pass, G., 2003. Preparation of serial sections of arthropods using 2,2-dimethoxypropane dehydration and epoxy resin embedding under vacuum. Biotechnic \& Histochemistry 78:1-5. https://doi. org/10.1080/10520290312120002

Qi, X., Ren, Y., Wang, X., 2017. New advances in the biodegradation of Poly(lactic) acid. International Biodeterioration and Biodegradation 117:215-223. https://doi. org/10.1016/j.ibiod.2017.01.010

Roth, J. A., Wilson, T. D., Sandig, M., 2015. The development of a virtual 3D model of the renal corpuscle from serial histological sections for E-learning environments. Anatomical Sciences Education 8:574-583. https://doi. org/10.1002/ase.1529

Royer, S. M., 1988. A simple method for collecting and mounting ribboned serial sections of epoxy embedded specimens. Biotechnic \& Histochemistry 63:23-26. https://doi. org/10.3109/10520298809107155

Ruthensteiner, B., 2008. Soft Part 3D visualization by serial sectioning and computer reconstruction. Zoosymposia 1:63-100. https://doi.org/1178-9913

Schoenwolf, G. C., Chandler, N. B., 1983. A Novel Sectioning Technique for Use in Descriptive Embryology: Bridging the Gap Between Paraffin and Plastic Serial Sections. The Anatomical Record 225:221-225. https://doi.org/10.1002/ ar.1092060211

Stephens, B., Azimi, P., El Orch, Z., Ramos, T., 2013. Ultrafine particle emissions from desktop 3D printers. Atmospheric Environment 79:334-339. https://doi.org/10.1016/j. atmosenv.2013.06.050

Tilic, E., von Döhren, J., Quast, B., Beckers, P., Bartolomaeus, T., 2015. Phylogenetic significance of chaetal arrangement and chaetogenesis in Maldanidae (Annelida). Zoomorphology 134:383-401. https://doi.org/10.1007/s00435-015-0272-9

Wali, N., Jagadeesh, J. M., 1988. A method for collecting semithin epoxy serial sections for light microscopy and 3-D reconstruction. Journal of Neuroscience Methods 23:9194. https://doi.org/10.1016/0165-0270(88)90179-3 